Itinéraires Itinéraires

Littérature, textes, cultures

\title{
Étant donnée : une fable poétique sur le régime de notre identité numérique
}

Étant donnée: A Poetic Tale about our Identity in the Digital Era

Cécile Portier

\section{OpenEdition}

Journals

Édition électronique

URL : http://journals.openedition.org/itineraires/3124

DOI : $10.4000 /$ itineraires.3124

ISSN : 2427-920X

Éditeur

Pléiade

Référence électronique

Cécile Portier, "Étant donnée : une fable poétique sur le régime de notre identité numérique 》,

Itinéraires [En ligne], 2015-3 | 2016, mis en ligne le 01 juillet 2016, consulté le 01 mai 2019. URL : http:// journals.openedition.org/itineraires/3124; DOI : 10.4000/itineraires.3124

Ce document a été généré automatiquement le 1 mai 2019.

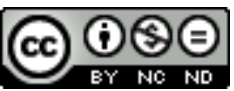

Itinéraires est mis à disposition selon les termes de la licence Creative Commons Attribution - Pas d'Utilisation Commerciale - Pas de Modification 4.0 International. 


\title{
Étant donnée : une fable poétique sur le régime de notre identité numérique
}

Étant donnée: A Poetic Tale about our Identity in the Digital Era

\author{
Cécile Portier
}

\section{NOTE DE L'ÉDITEUR}

L'ethos numérique, objet d'étude, devient sous l'impulsion de Cécile Portier un sujet de création par le biais d'un parcours à géométrie variable saisissant les traces invisibles et fortuites laissées ici et là pour en faire la matière d'une histoire. Cette reconfiguration interpelle et place en écho des points dissociés afin de proposer au lecteur de nouvelles clés de lecture à travers des " pièces ", des lieux, des identités multiples.

Si cette fiction poétique transmédia a pour point de départ l'œuvre de Marcel Duchamp, Étant donnés, artefact qui suggère au spectateur un jeu entre la visibilité, la transparence et l'absence d'évidence, cette fable propose d'autres éclairages qui s'aventurent du côté du politique et du littéraire. Ce texte présente cette expérience singulière de création, qui investit et questionne à la fois ce nouveau champ numérique.

1 Les données forment de plus en plus une nouvelle écriture du monde et de nous-mêmes. C'est même devenu l'écriture dominante. La question qui se pose aujourd'hui, c'est de savoir comment et par quoi nous sommes écrits, et qui détient les capacités à nous lire. Nos vies s'écrivent par traces, que nous laissons sans y penser, mais qui nous documentent et nous archivent, nous donnent aussi beaucoup à voir des autres et nous placent, alternativement, dans la position du voyeur ou du regardé mis à nu. Or, si nous sommes les premiers producteurs des traces numériques qui nous concernent, nous en sommes très peu auteurs. Les données produites à notre insu sont quantitativement plus importantes que celles que nous pensons maîtriser, dans un discours, une rhétorique consciente et personnelle. De plus, nous sommes des « auteurs » quasiment analphabètes : 
nous avons peu accès, nous ne savons que très peu lire les données qui nous définissent. Et pourtant, d'autres savent lire et exploiter ces données.

Même s'il est évident que la collecte des données personnelles à grande échelle n'a pas pour premier objectif de détailler telle ou telle vie individuelle, mais bien plus de dessiner massivement, statistiquement, des profils, retourner la situation fictivement et examiner ce que cette production massive a comme conséquence sur notre propre écriture de vie m'a semblé être un enjeu important.

3 La trace, dans l'acception commune (passée ?) est cette tension entre ce qui reste d'un temps qui n'est plus, ce qui insiste de ce temps révolu, et ce qui, de ce qui est passé et inscrit, est voué à s'effacer. La trace est une présence, qui est le témoignage de quelque chose qui n'est plus, d'une absence. À cela, à cette indécidable présence / absence, se substitue, dans le régime de la trace numérique, la notion d'une disponibilité infinie des inscriptions qui nous concernent et nous décrivent.

4 La trace est aussi cette sorte d'inscription qui pose d'une manière particulière le rapport des causes aux effets, de l'auteur à sa trace. Remonter à la source a toujours été une entreprise incertaine. Dans le régime de la trace numérique, cette incertitude n'existe plus ou si peu, les traces se remontent avec infaillibilité, jusqu'à des adresses, se recoupent. La trace était par définition ce qui pouvait se brouiller, elle devient l'élément clé de la traçabilité, ce régime d'écriture où le doute n'existe pas. Ce qui nous est refusé, dans cette nouvelle définition de la trace, c'est la possibilité d'inventer d'autres causes.

5 La trace est, était, ce qui vibre en permanence - comme une corde tendue entre deux pôles. Et c'est cette vibration (tension, ambivalence), qui est aujourd'hui circonvenue dans l'organisation de nos traces en données numériques. Se met en place une doctrine policière ou un marketing de la trace, produisant des dispositifs visant à l'assignation des intentions, à la réduction des incertitudes-quand la trace, justement, était une figuration de l'incertitude.

6 L'enjeu est donc littéraire, autant que politique : écrire pour réintroduire de l'incertitude dans nos traces, comme condition de notre liberté.

7 À partir d'un "prétexte » sur lequel nous allons revenir, se déploient donc dans cette fiction des textes et des propositions plastiques et interactives qui viennent interroger quelques-uns des dispositifs qui nous enregistrent, nous écrivent, et comment ces dispositifs viennent très profondément modifier notre rapport à notre intimité, et notre rapport aux autres et au monde.

8 Cet article en propose une visite guidée non exhaustive. Et cette visite commence par le titre.

\section{Étant donnée ${ }^{1}$ : un titre comme une citation, un détournement du champ}

9 Ce récit a pour point de départ l'œuvre célèbre de Marcel Duchamp, Étant donnés, où le spectateur regarde une femme nue, allongée, dont nous voyons tout, sauf le visage, et qui tient à la main un bec de gaz pour éclairer... le plein jour.

10 Nous sommes aujourd'hui très précisément dans cette situation : les données nous font la promesse de pouvoir tout voir, mais on oublierait assez facilement qu'il existe un point aveugle, que tout n'est pas si évident dans cette visibilité triomphante, et que peut-être il 
est nécessaire de venir éclairer le plein jour d'une autre lumière, poétique, politique, pour briser l'évidence du « tout est donné ».

11 Cette figure de la femme abandonnée, observée et éclairante dans son mystère même (en ce qu'elle fait voir ce qu'on ne voit plus à force de le trouver évident) sera le personnage de cette histoire. Elle viendra jeter une autre lumière sur ces données numériques qui ne se donnent pas tant à voir (car les données existent principalement sous le régime de l'invisibilité, en tout cas en ce qui concerne leur processus de collecte et de calcul dans des algorithmes que nous ne maîtrisons pas) mais travaillent à nous rendre chaque jour un peu plus « transparents».

12 Par ailleurs, cette femme nue proposée par Duchamp n'est pas un tableau, mais un artefact en trois dimensions, que nous sommes invités à regarder par un trou de palissade. Nous ne pouvons y coller qu'un œil, nous perdons donc toute idée de profondeur. Et effectivement, cela fait partie des craintes que nous pouvons avoir, qu'une description du monde hégémoniquement dominée par les données nous donne l'illusion de nous restituer le réel même, alors que nous n'aurions accès qu'à un monde plat, où la notion de point de vue serait bannie parce que balayée comme subjective, donc non légitime (alors que les chiffres, eux, parleraient « vrai »).

\section{Une entrée par l'image}

13 La navigation dans le dispositif part d'une promesse implicite qui nous est faite aujourd'hui sur de nombreux sites : celle de «surfer » sur des images, littéralement, de pouvoir rester à la surface sans être submergé par la déferlante des informations.

Car les données font image. Puisque l'information, proliférante, n'est plus humainement traitable, l'illusion se propage selon laquelle nous aurions moins besoin d'interpréter, de décrypter, de « lire » que de pouvoir visualiser, surfer, attraper. De façon inconsciente, ce rapport à la chose à connaître pourrait progressivement s'étendre à notre perception de tout le réel. À l'instar de Google Street View, le réel serait alors simplement réduit à l' image de ce qui est, et de façon si totale que cette représentation se suffirait à elle-même.

Dans ce site, nous allons donc surfer dans les images, selon cette même promesse, mais pour tomber sur la surprise... qu'elles recèlent du texte.

Quand on saisit l'adresse du site, www.etantdonnee.net, ce qui apparait en premier lieu c'est un écran blanc, puis la boussole de navigation de Google Street View descend comme un astre depuis le haut de l'écran, et vient s'imposer au beau milieu de la page.

Cette « molette ${ }^{2}$ » sera présente dans toute la navigation du site : c'est par elle que l'on se déplacera dans l'histoire, de chapitre en chapitre par les quatre points cardinaux, et linéairement selon la narration, en cliquant sur le petit signe " plus » situé en dessous. On ne tourne plus de pages, on se déplace comme sur un plan. Puis le titre apparait, mais comme perturbé par un brouillage, et accompagné de ce sous-titre, qui est comme le contre-programme de ce projet : le monde n'est plus lisible, il est navigable.

Le petit signe "plus " présent en dessous de la boussole se met à clignoter pour nous inciter à zoomer. 


\section{Chapitre I: Apparition}

19 En cliquant, en zoomant, nous sommes emmenés devant un drôle de paysage, une photographie de terrain vague (capture d'écran réalisée depuis Google Street View) : c'est le lieu de la scène. De quelle scène, nous ne le savons pas encore. Nous savons seulement cela, que nous sommes dans un espace indéfini, aux marges, un endroit où l'on n'est pas censé aller et qu'il est donc intéressant de visiter, comme une sorte d'inspection de l'envers du décor.

20 L'image passe ensuite en noir et blanc, n'est plus qu'un dessin, puis se recolorise, mais sans atteindre au même niveau de « réalité » qu'au début. Nous savons que nous sommes entrés dans la fiction.

21 Puis, se produit un mouvement de zoom vers une zone d'herbe où semble reposer quelque chose...

http://etantdonnee.net/\#apparition

Nous découvrons un corps de femme, nu, endormi. Étant donnée commence comme un polar, mais c'est une fable, qui doit plus à La Belle au bois dormant ou à Peau d'Âne qu'au Dahlia noir, nous allons le constater.

Inexorable, cette pièce interactive d'Alexandra Saemmer, joue de la nudité et de sa visibilité dans un très exact contrepoint, par sa posture, de celles de L'Origine du monde de Courbet, de l'Étant donnés de Marcel Duchamp, et bien sûr de l'imagerie commune de la pornographie. La promesse du tout est à voir est ici déniée d'emblée. Alors que dans les archétypes cités, seul le visage est dérobé pour mieux mettre en scène le sexe de la femme, ici, ni le visage ni le sexe ne sont visibles. Reste seulement l'idée d'une femme nue, et la frustration de ne pas en savoir plus. Puis, la silhouette disparaît, lentement, comme évanescente. Elle est son propre mystère.

Elle chuchote, et nous pouvons agir sur l'image. Quand on promène la souris sur l'endroit où était son corps, des pixels de chair apparaissent, puis s'évanouissent. Aussi vite que nous allions à balayer cette surface, à la caresser ainsi, nous n'arrivons jamais à la reconstituer, à la rendre de nouveau disponible.

http://etantdonnee.net/\#inexorable

Une femme donc, offerte dans sa nudité au regard unidirectionnel des données. Tout comme dans l'installation de Marcel Duchamp où le regard par le trou de la serrure, et donc par un seul œil, enlève tout relief à la scène, regarder les personnes uniquement par les données produites par elles ou pour elles produit l'illusion de tout voir car l'intimité est offerte, mais fait oublier qu'il y a un hors-champ et aussi une profondeur de champ qui échappent à cette vision en un seul point de vue.

http://etantdonnee.net/\#expose

La proposition de départ de cette fiction est simple, et formulée comme le début d'un problème mathématique: étant donnée une femme que vous, lecteur, égaré dans ce terrain vague, retrouvez nue et inconsciente dans un terrain vague. Aucune blessure sur elle, aucun signe de son identité. Elle est comme un fichier vierge posé dans la ville. Sa présence est comme une équation à résoudre, non pas tellement celle de savoir pourquoi elle apparaît, mais pourquoi elle est déplacée. 
Votre regard zoome encore, vous tombez sur son corps, sa nudité, l'incongruité de sa nudité vous fait peur, sa fragilité. Vous appelez les secours, vous la recouvrez. Premier acte par lequel vous apposez sur elle quelque chose, une seconde peau, comme si la sienne propre ne suffisait pas à sa survie.

http://etantdonnee.net/\#couverture_de_survie

\section{Chapitre II - Identification}

Et puisqu'il s'agit de peau, c'est à cet endroit même que vous allez la lire. Cette femme est emmenée à l'hôpital et on fait parler son corps inconscient en le branchant à des machines qui commencent à l'enregistrer, l'évaluer, le placer dans des normes. On le sait, l'enjeu du corps et de la santé est central dans le traitement des données : qu'on pense au marché des assurances, que le quantified-self ${ }^{3}$ et les dispositifs qui le facilitent, comme l'Apple Watch, vont profondément modifier. C'est un des premiers milieux où se perçoit le plus nettement le risque que la personne soit objectivée dans une prestation (de soin, en l'occurrence) qui n'a plus grand-chose d'une relation.

http://etantdonnee.net/\#identification

À part ses «faibles scores ", qui parlent à sa place, ce corps s'exprime seulement par sa respiration, avec cette œuvre d'Alexandra Loewe faisant écho encore une fois dans son titre à Courbet: Beyond Abstract: The Origin of the World. Sur la vidéo, on voit seulement un drap d'hôpital, comme déchiré, et qui s'entrouvre sur d'autres ouvertures encore, tandis qu'un souffle de plus en plus péniblement émis se fait à peine entendre.

http://etantdonnee.net/\#beyond_abstract

Ce corps s'exprime aussi par sa palpitation, avec cette interrogation, « Où est mon sang? » sur ce qui est encore vital, dans ce flux déjà important de chiffres que ce sang charrie.
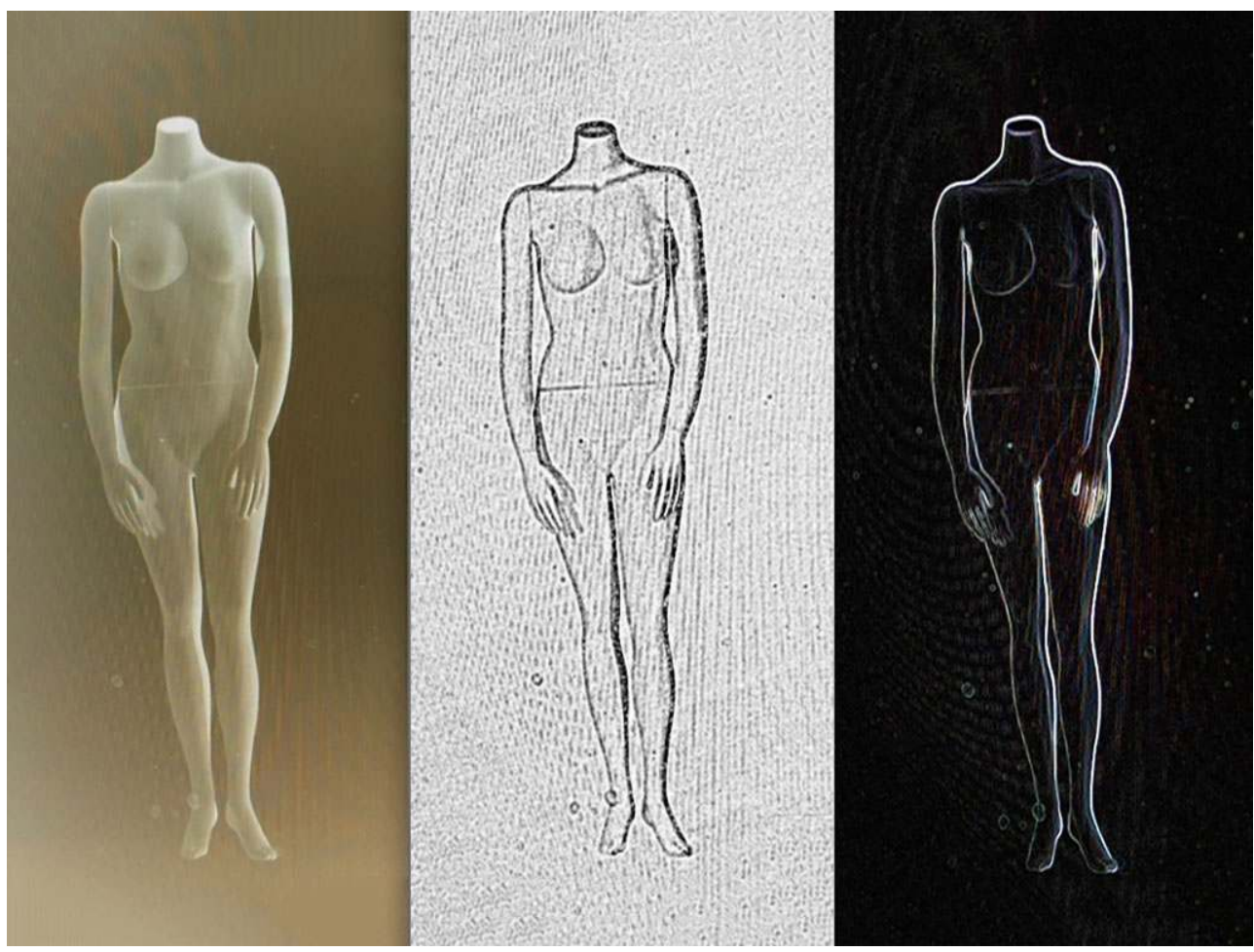

http://etantdonnee.net/\#patiente_x 
reconnaissance faciale, qui peuvent être désormais directement embarqués dans les
smartphones ou les Google glass, et donner de nous une réalité augmentée, une sorte
d'épiphanie de nous-mêmes, d'où nous ressortons tout auréolés de notre existence sociale (c'est-à-dire celle que nous déployons sur les réseaux sociaux). 

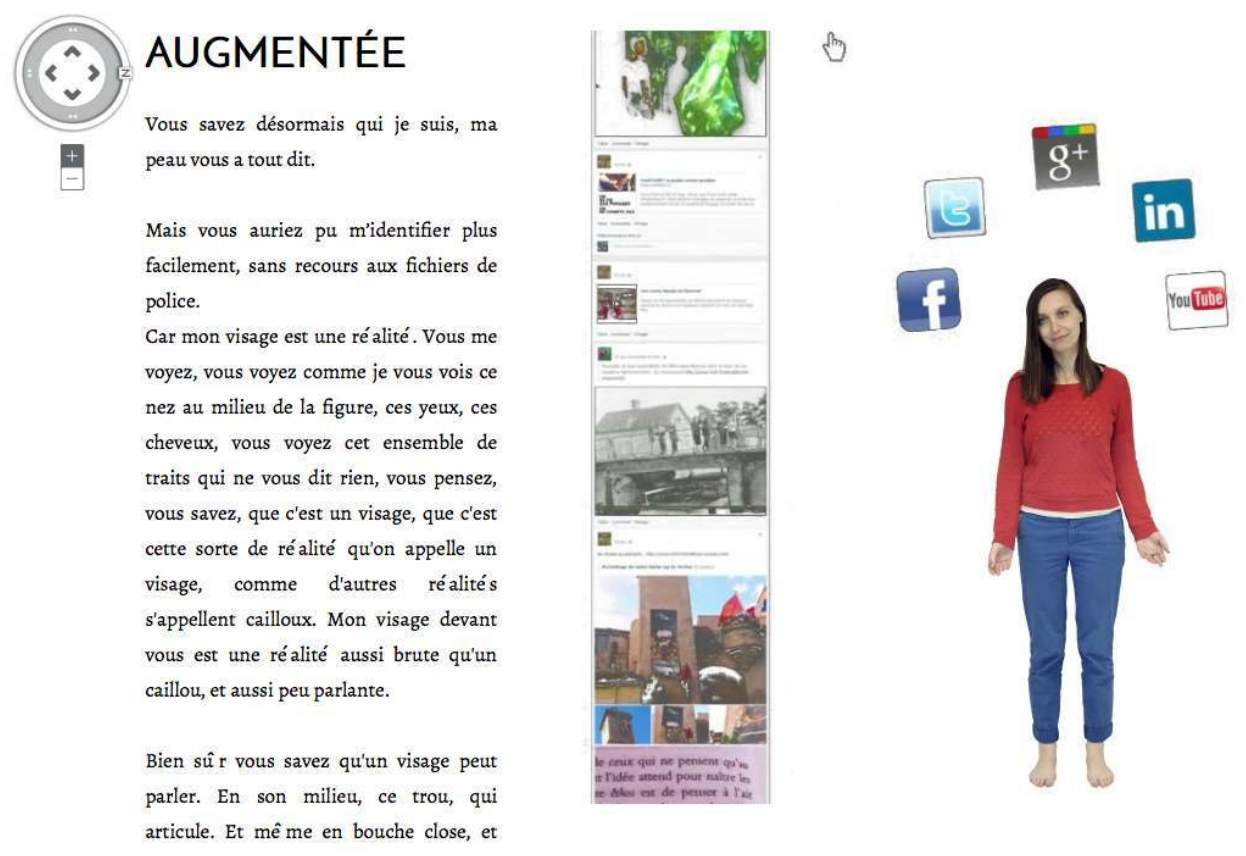

http://etantdonnee.net/\#augmentee

Maintenant que vous, lecteur, savez qui est cette femme, vous allez vouloir la rhabiller de toute sa vie en recollectant l'ensemble des données qu'elle a disséminées avant sa disparition.

Vous allez donc partir à la recherche de ses traces antérieures, pour lui «redonner » sa mémoire. Elle, tout au long de l'histoire, se laissera faire, restera comme inerte. Dans le même temps, elle ne délivrera rien. Vous saurez que nous savons, que vous savez d'elle de plus en plus de choses, que vous en savez tout, mais le récit lui-même ne dira jamais rien de concret sur ce personnage, qui continuera d'être ce spectre, rajoutant machinalement des voiles contre la transparence dont vous voulez l'affubler. (C'est en cela que sert d'avoir pris, pour cette histoire, un personnage féminin : pour ce que son imagerie joue déjà en permanence avec la notion de l'intime à préserver, voiler, violer.)

http://etantdonnee.net/\#interlude_1

39 Cette histoire commence donc comme un polar et très rapidement s'éloigne du genre: rien n'est effectivement résolu, ou plus exactement, nous savons qu'il y a des résolutions, mais elles nous sont comme inaccessibles, et surtout inopérantes, si la question que nous avions concernant cette femme était, non pas de savoir tout d'elle, mais de la connaître vraiment, au-delà de ses comportements.

40 Et c'est dans cet infime et pourtant gigantesque écart que se situe peut-être notre liberté, en tant que personne, face à des processus d'enregistrement et de traitement de nos traces si puissants qu'ils peuvent en effet savoir jusqu'à quoi nous pensons, mais qui sont par ailleurs incapables de rentrer avec nous dans une relation autre que celle de prédation (mais le crocodile connait-il sa proie ?).

\section{Chapitre III - Recollection}

41 Ce chapitre, central dans la fiction, est placé sous le signe du nuage. Le nuage est une nouvelle modélisation de notre rapport à l'espace et au temps. C'est un lieu qui n'en est 
pas un, toujours mobile, labile, irrattrapable, mais qui peut quand même nous pleuvoir dessus. C'est la figuration de l'ailleurs. Et c'est dans cet ailleurs que nous nous stockons désormais, que nous stockons notre mémoire, et avec elle, tous nos possibles ${ }^{4}$. Cette femme, ce personnage, s'adresse de nouveau à vous pour dire cela: "Ma mémoire est ailleurs, vous pouvez la trouver sans moi. Et vous verrez, comme une pluie, apparaîtra le texte de ma vie.»

Mais pour cela, il faut détenir quelques clés, même si une bonne partie de notre mémoire est en accès libre et public. Nos logins (identifiants et code d'accès) sont essentiels, pour nous percer à jour. Qui les détient entre en nous. À ce moment de l'histoire, le lecteur (navigateur? il est vrai qu'on ne lui propose pas seulement de lire), pourra manipuler grâce au plugin Sketchfab une modélisation 3D du personnage d'Étant donnée, dont la peau change d'aspect, tour à tour simple structure filaire, plâtre neutre ou bronze de statue : elle est entièrement zoomable, retournable, et on peut la voir sous toutes les coutures. Or, des coutures, elle en a : les défauts du scan 3D, fait à bas coût avec le périphérique Kinect, ont eu pour effet de faire opportunément de cette modélisation un genre de colosse aux pieds d'argile (les chevilles sont absentes), et surtout, de lui laisser le crâne ouvert, si bien qu'on pourrait presque la retourner comme un gant. À tout le moins visiter son vide intérieur. Et cette femme peut nous dire alors: «J'entre avec vous dans ma propre mémoire. " On retrouve ici, imagée, une partie de la problématique développée par Olivier Ertzscheid (2009) : la volumétrie considérable des données produites par et sur les individus, notamment par les logiques déclaratives encouragées sur les réseaux sociaux, autorise toute sorte d'exploitations, à des fins d'études ou de commercialisation. La personne n'est plus propriétaire de son identité. Elle est comme fouillée, et définie de l'extérieur.

43 Après avoir forcé les logins de cette femme et inspecté son intérieur (jusqu'à jouer la scène d'un Google Street View qui inspecterait son appartement, dans une pièce de Juliette Mézenc et Stéphane Gantelet, Go home view, qui forme un moment de cette histoire), vous, lecteur, vous assistez à l'inventaire de ses archives personnelles. Réalisé en détournant le logiciel de présentation de conférence Prezi, cet inventaire «balaie» le contenu d'un bureau d'ordinateur, pour s'apercevoir qu'il n'y a rien de consistant à cet endroit, que tout est contenu plus haut, plus loin, dans des espaces indéfiniment gigognes, que la mémoire est au clou, au cloud, et que ce nuage ressemble à s'y méprendre à un écran de fumée.

Alors, si rien n'est détenu, à part dans les nuages de la délégation, peut-on savoir qui est cette femme par le seul contenu de son ordinateur? Le moment "Je reçois, donc je suis ", propose la lecture par une voix synthétique d'une très longue liste de spams reçus. Sont seulement énoncés, les uns à la suite des autres, les «objets " des messages, comme autant de promesses, de propositions, qui nous sont adressées, et, qui par contextualisation et ciblage des profils formeraient une sorte de portrait en creux, de moulage de notre personnalité, de nos préoccupations.

Bien entendu, si la publicité contextuelle et le ciblage existent bel et bien, les spams dans leur immense volume constituent des adresses aveugles, donnant ensemble de qui les reçoit un portrait chinois à la fois grotesque et invraisemblable. Néanmoins, si nous résumons, qu'en ressort-il ? Celui qui reçoit cela, celui qui est donc ce qu'il reçoit, est un personnage crédule, atteint d'une incapacité à désirer et à jouir, souffrant d'insomnie, miné par la peur, les problèmes financiers, la solitude et l'ennui. Est-ce cela notre miroir, ou la fabrication sociale de notre devenir? 
cecdotique, le véritable travail sur la langue souterrainement à l'œuvre dans les configurations des algorithmes des $\mathrm{GAFA}^{6}$, lui, ne l'est pas, comme le montre par exemple cet article de Frédéric Kaplan (2012), « Nos langues à l'heure du capitalisme linguistique ». Non seulement nous pouvons constater la mise en œuvre d'une régularisation de la langue (nous orienter vers tel mot plutôt que tel autre), mais aussi, au fur et à mesure que cette régularisation s'opère, vers une prédictibilité de plus en plus grande de nos propres discours. La question de la mise à nu de nos intentions est aujourd'hui au cœur des débats soulevés par les révélations d'Edward Snowden sur la surveillance de masse des internets par la NSA', mais aussi, dans le domaine marketing, par l'utilisation des big data par les entreprises. La Théorie du drone de Grégoire Chamayou (2013) montre comment peuvent être dessinés, à partir de nos traces, des " pattern of life ", d'où peuvent être déduits nos comportements futurs.

Que reste-t-il alors de nos intimités dans ces descriptions invasives de nos vies? Que reste-t-il de notre liberté face aux prédictions des algorithmes? La pièce «Inter(faces)» vient clore ce troisième chapitre de la recollection par cette question : face à l'écran, face à l'œil machinique de la caméra, face au miroir tendu par l'algorithme, y a-t-il (encore) quelqu'un? Ce texte est lisible en trois moments, l'écran, l'œil, le miroir, chacun symbolisés par une "version» du personnage, tournant à la manière des bandits manchots de casino jusqu'à s'arrêter sur l'une ou l'autre des figures et des versions du texte, dans un hasard de "gagne-toujours", mais quoi ? On pense ici à cette phrase de Jean Cocteau : «Les miroirs feraient bien de réfléchir un peu plus avant de renvoyer les images 8 .» De fait, les caméras deviennent des miroirs à mémoire, là où les miroirs reflétaient, les caméras enregistrent, puis les logiciels traquent, décodent, interprètent. 
Et toute cette puissance de calcul ne produit aucune réflexion, seulement des solutions, comme l'on dit bien sûr au sens mathématique, mais aussi au sens chimique - décomposition d'une complexité en éléments simples et homogènes : une multitude de données, totalement étrangères à l'énigme de la personne dont elles proviennent. Ainsi, la subjectivité ne finit-elle pas par s'effacer devant ce qui la traque?

\section{Chapitre IV - Disparition}

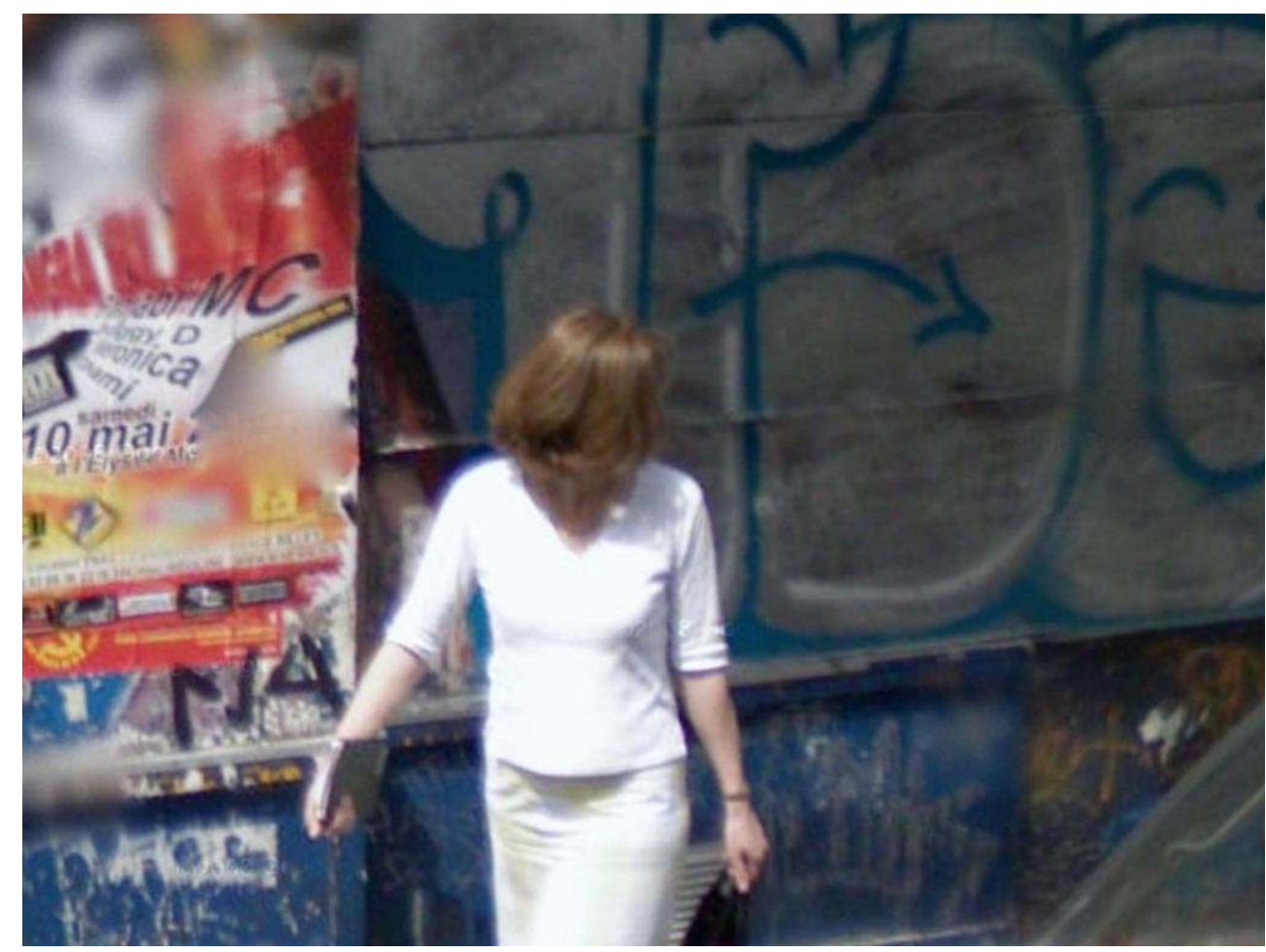

http://etantdonnee.net/\#disparition

Et si notre liberté se logeait dans cette sorte d'oubli, de méconnaissance de soi-même qui frappe le personnage de cette fiction? Vous qui la lisez, qui l'observez, qui savez tout d'elle, ou croyez le savoir, allez pouvoir retourner en flash-back dans sa vie jusqu'au moment où elle en a disparu.

Et là, vous allez découvrir qu'elle a justement disparu pour tenter d'échapper à sa description. Elle s'est comme détachée de ce qui progressivement la cernait de plus en plus, s'en est arrachée, au risque d'y laisser « sa peau », cette peau de données qu'on lui a écrite sur le dos (à la manière dont le châtiment s'écrit sur la peau du condamné dans $L a$ Colonie pénitentiaire de Kafka). Vous assistez à tout le processus ayant précédé sa disparition : au départ, elle est cette femme de valeur, qui compte et sait compter. Elle est totalement adaptée. Mais cette obsession du comptage prend toute la place, et lui tient lieu de seul rapport au monde. Elle est entièrement soumise à la logique spéculative qui en découle, ne vivant sa vie que dans le rapport à la ligne morte (traduction littérale, empreinte d'une inquiétante étrangeté, de la très commune dead line à laquelle se cognent régulièrement nos actes). Pour ce moment de l'histoire, la vidéo qu'a réalisée Laure Chapalain pour accompagner le texte opère un montage entre une femme courant un marathon et des images de «flash crash $^{9}$ » boursiers, rappelant que l'essentiel des 
transactions est aujourd'hui réalisé par des ordinateurs et que le courtage à "haute fréquence", où chaque microseconde compte, est devenu la norme. Dans ce monde d'interfaces où il n'est pas sûr qu'il y ait encore quelqu'un, tout est matière à spéculation, même le temps. suiviez, vous la teniez. Elle était dans le viseur d'une caméra de vidéo surveillance, et subitement, elle sort du champ, et n'est «reprise» dans aucun autre dispositif. Elle s'échappe, en d'infinies possibilités isochrones. Son existence, comme un liquide trop longtemps contenu et tout d'un coup déversé, est en expansion sur la carte.

http://etantdonnee.net/\#expansion

Elle devient un personnage quantique : si on clique sur sa localisation, aussitôt le "Vous êtes ici " s'échappe et se place aléatoirement ailleurs sur l'écran. Cette femme est désormais où bon lui semble et à tous les vents, on ne sait pas son chemin. On sait seulement qu'elle marche, et que sous ses pas devenus libres la ville s'invente et s'écrit différemment de nos chemins d'habitude. Elle est sortie du «tout est déjà écrit ». Notre modernité avait troqué de ce point de vue les fatums, les destinées, pour de bien plus prosaïques prévisibilités des parcours de vie, déduites des actes et comportements passés. C'est à ce nouveau fatalisme qu'elle échappe, par son imprévisibilité. C'est bien dans cet ailleurs qu'elle devient un objet littéraire intéressant. Car on aimerait bien rendre compte de tous ces possibles qu'elle invente ainsi. Mais comment le faire, quand le point de vue omniscient a disparu?

http://etantdonnee.net/\#la_ville_est_sous_mes_pas

Cette errance est donc racontée sous la forme d'une sorte de cadavre exquis, combinaison aléatoire sans cesse recommencée entre des textes d'auteurs ou d'internautes inconnus racontant chacun avec leurs mots, leur sensibilité, un moment de la déambulation de cette femme. Ce chemin n'est d'ailleurs pas que d'écriture : une combinaison, aléatoire elle aussi, de morceaux de villes venus du monde entier, attrapés via Google Earth, vient former la carte sans cesse renouvelée de cette errance collective, et par définition impossible à terminer. Manière de rejouer à l'heure numérique une sorte de dérive psycho-géographique collective, pour reprendre à plusieurs, à tous, le choix du chemin. 
61 Ce récit n'est donc pas seulement le constat d'une aliénation, celle de cette femme, si bien décrite par les données, que l'idée même d'intériorité devient superfétatoire. À partir de cet oubli qui désormais seul la constitue, le personnage d'Étant donnée peut se réinventer. Et le faire avec nous tous, qui la lisons, et qui aussi l'écrivons, mais cette fois-ci, comme de l'intérieur, comme si c'était nous, comme si c'était de notre propre liberté (de vie, d'écriture) qu'il s'agissait.

Vous pouvez désormais mieux comprendre où, et comment elle a disparu. Une vidéo vous propose de vous redonner le réel même, à savoir Google Street View, qui se positionne dans sa description du monde comme la fameuse carte de Borges à l'échelle 1/1 (Borges 1951), et d'aller fouiller par vous-même, retrouver la disparue. Et vous la localisez enfin, arrêtée juste avant d'entrer dans une voie non couverte par Google Street View. Elle est allée jusqu'à ce nouveau « bord du monde connu », elle a sauté.

http://etantdonnee.net/\#l_angle_mort_est_mon_horizon

Or, cette voie qu'elle a suivie, c'est le Passage du désir à Paris, dans les faits une sombre voie privée, coincée entre la rue du Faubourg Saint-Denis et le Boulevard Sébastopol, une rue très peu avenante donc, à peine une rue, mais qui a l'avantage d'être décrite comme une voie malgré tout dans Google Street View (une flèche nous indique qu'on peut y aller), mais d'être trop étroite pour que la Google car s'y faufile. Aussi, quand on cherche à s'y introduire, on en est tout de suite éjecté, par l'autre côté. Nous sommes face à un espace inconnaissable.

Le personnage d'Étant donnée a ainsi disparu dans un trou noir, un trou noir de données où toute image s'absente, où toute description extérieure est annulée, remplacée par la vie vécue pour elle-même, écrite par elle-même: ainsi pourrait-on donner une description de ce qu'est le " passage du désir ».

Je voudrais terminer l'évocation de ce récit en citant ces derniers mots :

Pour qu'un corps puisse s'échapper de l'attraction d'un astre, il faut qu'il acquière une certaine vitesse. Plus l'astre est dense, plus cette vitesse devra être élevée. Un trou noir est un astre dont la vitesse de libération est supérieure à la vitesse de la lumière.

Le désir est un astre dont la vitesse de libération est supérieure à celle des images.

La surface du trou noir, la science l'a nommée "horizon des événements». Cet horizon des événements est indépassable. Aucun astre constitué en trou noir ne laisse voir l'en deçà, l'issue finale de son effondrement sur lui-même. Sauf que. Sauf cela, qu'il existe l'hypothèse de l'existence de petits morceaux d'espace-temps dont la capacité d'attraction serait infinie, et qui seraient dépourvus pourtant de surface d'invisibilité, de peau, d'horizon des événements. Cette aberration, qui n'est qu'une hypothèse, porte le nom de singularité nue.

Une singularité nue c'est cela : un lieu d'attraction, de perdition, de révélation. C'est le lieu dont votre regard ne sait rien, bien que tout soit donné à voir. C'est l'endroit où la disparition est une obscénité.

C'est le lieu de votre désir, autant dire de votre âme. C'est le peep show de votre propre mort.

C'est le lieu de l'écriture, et de quoi d'autre ${ }^{10}$ ?

Tout est connu, consommé, et cette femme revient à son opacité. Tout peut recommencer. Il suffit pour cela d'actionner de nouveau les points cardinaux de la molette de navigation, on retrouvera le corps nu, l'apparition, l'énigme, comme une question indéfiniment posée à nous-mêmes : quel est notre désir, c'est-à-dire, notre résistance ${ }^{11}$ ? 


\section{BIBLIOGRAPHIE}

Borges, Jorge Luis, [1951] 1994, « De la rigueur de la science », dans Histoire universelle de

l'infamie / Histoire de l'éternité, Paris, 10/18.

Chamayou, Grégoire, 2013, Théorie du drone, Paris, La Fabrique éditions.

Ertzscheid, Olivier, 2009, «L'homme est un document comme les autres : du World Wide Web au World Life Web », Hermès, CNRS-Editions, p. 33-40, [En ligne], <sic 00377457v2>.

Kaplan, Frédéric, 2012, "Nos langues à l'heure du capitalisme linguistique », internetactu.net, 6 avril 2012, http://www.internetactu.net/2012/04/06/nos-langues-a-lheure-du-capitalismelinguistique/.

Portier, Cécile, 2011, «Écrire s’adresse », remue.net, 19 janvier 2011, http://remue.net/spip.php? article4044.

\section{NOTES}

1. Artistes et auteurs contributeurs : Laure Chapalain, Emmanuel Cohen, Benjamin Dufour, Alain François, Stéphane Gantelet, Thomas Guillaud-Bataille, Alexandra Loewe, Pierre Ménard, Juliette Mezenc, Julien Pannetier, Pascale Petit, Alexandra Saemmer. Graphisme: Laure Chapalain; développement : Julien Kirch)

2. Cette boussole sans points cardinaux mentionnés indique des directions, des lignes d'orientation.

3. Le quantified-self (en français, "mesure de soi ») est un "mouvement qui regroupe les outils, les principes et les méthodes permettant à chacun de mesurer ses données personnelles, de les analyser et de les partager. Les outils du quantified-self peuvent être des objets connectés, des applications mobiles ou des applications web» (Wikipédia, l'encyclopédie libre, http:// fr.wikipedia.org/w/index.php?title=Quantified_self\&oldid=124717849).

4. Le cloud computing ou l'informatique en nuage est « l'exploitation de la puissance de calcul ou de stockage de serveurs distants par l'intermédiaire d'un réseau " (Wikipédia, l'encyclopédie libre, http://fr.wikipedia.org/w/index.php?title=Cloud_computing\&oldid=127059767).

5. La guematria est une méthode d'interprétation biblique établissant une correspondance entre les lettres, mots et versets de la Torah d'une part, et des nombres d'autre part.

6. L'acronyme GAFA désigne les quatre grandes firmes américaines dominantes sur le marché du numérique : Google, Apple, Facebook, Amazon.

7. National Security Agency, «organisme gouvernemental américain responsable du renseignement d'origine électromagnétique et de la sécurité des systèmes d'information et de traitement des données du gouvernement américain " (Wikipédia, l'encyclopédie libre, http:// fr.wikipedia.org/w/index.php?title=National_Security_Agency\&oldid=127027775).

8. Voix off dans le film Le Sang d'un poète (1930), Jean Cocteau.

9. Le Trading haute fréquence désigne l'ensemble des technologies disponibles permettant d'exécuter automatiquement des ordres boursiers, en volumes importants et à très grande vitesse. Les prises de position en découlant ne sont pas destinées à être conservées au-delà de quelques micro-secondes. Cette volatilité exacerbée est à l'origine de quelques effondrements 
boursiers éclairs. Le plus spectaculaire, en mai 2010, a vu l'indice Dow Jones perdre 1000 points et en regagner 600 en 10 minutes.

10. http://etantdonnee.net/\#l_angle_mort_est_mon_horizon.

11. Cécile Portier, «Écrire s'adresse », 19 janvier 2011, http://remue.net/spip.php?article4044.

\section{RÉSUMÉS}

Étant donnée est une fiction web inspirée de l'œuvre célèbre de Marcel Duchamp, Étant donnés. Une femme est retrouvée, amnésique: elle ne se connaît plus. Par les données, on peut l'identifier, tout retrouver. Dans cet article, Cécile Portier, initiatrice de cette fiction à laquelle elle a convié d'autres auteurs et artistes, en propose une visite guidée. Les différentes "pièces » du site web sont les étapes successives d'une enquête angoissante, où l'on comprend que l'identité semble ne plus avoir besoin de s'étayer sur une intériorité. Tout est donné, tout est transparent. Mais quelque chose est à déjouer de ce nouveau régime d'existence où les données écrivent à notre place, et la littérature peut y jouer son rôle.

Étant donnée is a web fiction inspired by the famous work of Marcel Duchamp, Étant donnés. A woman is discovered, she is amnesiac, doesn't even know who she is. The data can help naming her, and finding again her whole life. Cécile Portier wrote this fiction and invited other writers and artists to participate. In this paper, she proposes a sort of "guided tour" of the story. The different parts of the website are the successive steps of a worrying inquiry, because we progressively understand that pricacy, individual subjectivity, is no longer necessary to define someone's identity. Everything is already known and written. But the story suggests that it is possible to withstand this pressure of the data, and that litterature, perhaps, can help regain control on our lifes.

\section{INDEX}

Mots-clés : données, traces numériques, surveillance, mémoire, problématique de l'identité, intimité, subjectivité, autonomie, écriture

Keywords : data, digital tracks, identity issues, memory, privacy, subjectivity, independance, writing

\section{AUTEUR}

\section{CÉCILE PORTIER}

Cécile Portier mène une activité d'écriture où les formes papier, numérique et performance se côtoient. Elle a fait paraître trois ouvrages : Contact (Éditions du Seuil, collection « Déplacements » dirigée par François Bon), en avril 2008, Saphir Antalgos, travaux de terrassement du rêve (Publie.net), en janvier 2010, et Les Longs silences (Publie.net), en novembre 2015. Elle tient un blog, http://www.petiteracine.net, où, à travers différents projets conçus comme des chroniques 
où images et textes se répondent, elle s'attache à explorer comment s'articulent aujourd'hui le social et l'intime. 